\title{
COMPARAÇÃO ENTRE OS PERFIS DE LIBERAÇÃO DE AZUL DE METILENO A PARTIR DE MICROPARTÍCULAS DE ALGINATO-QUITOSANA EM PH ÁCIDO
}

\author{
METHYLENE BLUE LOADED ALGINATE-CHITOSAN MICROPARTICLES \\ RELEASE PROFILE COMPARISON IN ACID PH
}

\author{
FUJIWARA, G. M1'; KAMINSKI, G. T. A².; FIN, M. T.2; SASSO, D. G. B. ${ }^{2} ;$ TIBERIO, C. C. ${ }^{2}$; \\ MIGUEL, M. D. ${ }^{3}$; ZANIN, S. M. W. ${ }^{3}$ \\ 1 - Mestranda em Ciências Farmacêuticas - Universidade Federal do Paraná \\ 2 - Acadêmicos do curso de Farmácia - Universidade Federal do Paraná \\ 3 - Docentes do curso de Farmácia - Universidade Federal do Paraná \\ Email para contato: sandrazanin@ufpr.br \\ REC: 05/2010 AC: 06/2010
}

\begin{abstract}
RESUMO
A utilização de alginato de sódio como biopolímero formador de micropartículas vêm sendo largamente estudada e investigada para obtenção de formas farmacêuticas de liberação controlada. Neste trabalho avaliou-se o perfil de liberação do azul de metileno de micropartículas deste polímero, preparadas por reticulação iônica com quitosana, com a presença ou não de sódio, em tampão ácido clorídrico $\mathrm{pH} 1,5$, considerando o azul de metileno como modelo de princípio ativo de baixo peso molecular.
\end{abstract}

Palavras-chave: Microparticulas. Alginato de sódio. Quitosana. Perfil de liberação.

\begin{abstract}
Alginate microparticles have been extensively studied to obtain sustained release pharmaceutical forms. The release profile of methylene blue, as low molecular weight drug standard in alginate microparticles, was evaluated in $\mathrm{pH} 1.5$ buffer. The presence of sodium was analysed in the formulations.
\end{abstract}

Keywords: Alginate microparticles. Sodium alginate. Chitosan. Release profile.

\section{INTRODUÇÃO}

O controle de liberação de algumas substâncias, como fármacos, corantes, pesticidas e aromatizantes, possui relação estreita com a tecnologia dos sistemas matriciais e reservatório poliméricos, amplamente empregados na forma de micropartículas em indústrias farmacêutica, cosmética e alimentícia (SUAVE et. al., 2006; TAN; CHANG e HENG, 2009).

Micropartículas de alginato são usualmente feitas por gelificação iônica, de processo simples, no qual uma solução de alginato contendo a substância a ser encapsulada é gotejada sobre uma solução iônica em concentrações variáveis. A microencapsulação consiste no empacotamento de pequenas quantidades de substâncias sólidas, líquidas ou gasosas por uma fina membrana polimérica, visando reduzir sua reatividade a fatores não favoráveis, como luz, oxigênio, umidade, temperatura e contato com outras substâncias incompatíveis, podendo ainda controlar a liberação ou perda da substância por evaporação, podendo também facilitar a manipulação da 
mesma (SANTOS; FERREIRA e GROSSO, 2000; ALLEN JÚNIOR; POPOVICH e ANSEL, 2007; CORRÊA, 2008).

A liberação controlada de fármacos através das micropartículas é um processo relacionado às formas farmacêuticas com liberação modificada, a qual pode ser retardada, quando a liberação do fármaco não se inicia logo após sua administração, ou prolongada, quando existe liberação gradual do fármaco, o que reduz sua frequência de tomada (SUAVE et al., 2006; STORPIRTIS et al., 2009).

O controle da liberação do material encapsulado está diretamente relacionado ao material encapsulante, estrutura química, espessura, porosidade e solubilidade. O tamanho e a distribuição dos poros nas micropartículas influenciam a difusão de moléculas através da rede de gel, sendo que partículas mais porosas permitem liberação mais rápida do material encapsulado (CORRÊA, 2008).

O uso do alginato de sódio como material encapsulante é amplamente explorado, por ser um polímero natural de relativo baixo custo, de fácil obtenção, biocompatível, atóxico e biodegradável, que não necessita utilização de solventes orgânicos e nem condições rigorosas de temperatura para a formação das partículas (FUNDUEANU et al., 1998; SCHOUBBEN et al., 2010).

O alginato é um polímero linear obtido de algas marinhas marrons, constituídos de duas unidades monoméricas, ácido gulurônico e o manurônico. Em solução aquosa, o alginato de sódio é solúvel, porém, quando em contato com soluções contendo íons divalentes, como o $\mathrm{Ca}^{+2} \mathrm{e} \circ \mathrm{Mg}^{+2}$, torna-se insolúvel devido a reticulação iônica dos grupamentos carboxila com os íons, formando uma rede de gel estável, em configuração de caixa de ovo (TU et al., 2005; DARRABIE; KENDALL e OPARA, 2006; HAN et al., 2007; DOHNAL; STEPANEK, 2010) .

As micropartículas de alginato-Ca são muito utilizadas para encapsulação de macromoléculas instáveis em água, como as proteínas, pois criam um ambiente hidrofílico que dificulta sua desnaturação (SCHOUBBEN et al., 2010). Estas partículas são utilizadas para encapsular materiais bioativos, devido a grande porosidade da membrana, que permite a passagem de pequenas moléculas, mas não as de água, sendo também inapropriada para moléculas de baixo peso molecular (FUNDUEANU et al., 1998; LOPEZ e ALONSO, 2001). A porosidade dessas micropartículas determinará a quantidade de material retido e o tempo de liberação do mesmo, sendo que em geral, a liberação do material encapsulado em alginato é normalmente rápida. Uma das maneiras de aumentar o controle desta liberação é utilizar um polímero carregado positivamente para formação de uma camada protetora, como a quitosana, que irá diminuir a porosidade das partículas através da interação iônica (TU et al., 2005; LIRA et al., 2009; DOHNAL e STEPANEK, 2010).

A quitosana é um polímero que ocorre naturalmente em alguns microrganismos, mas que também pode ser resultante da desacetilação da quitina. É um também um biopolímero biodegradável, muito utilizado na indústria farmacêutica como adjuvante em formulações sólidas de uso oral, atuando na liberação controlada de fármacos e melhorando a dissolução e biodisponibilidade de fármacos insolúveis em água (LOURENÇO, 2006).

A associação de alginato-quitosana mostrou-se interessante quando apresentou melhora na mucoadesividade do alginato, sendo promissor seu uso em sistemas de liberação mucoadesivo, principalmente a gástrica (TU et al., 2005). 
Gaserod, Smidsrod e Skjask-Braek (1998) e Tu et al. (2005), sugeriram a introdução de sódio no processo de obtenção das micropartículas para melhorar a retenção e liberação de princípio ativo.

A liberação do material encapsulado pode ocorrer por difusão do material encapsulado pela rede polimérica, por rompimento mecânico das partículas, ou ainda, por meios $\mathrm{pH}$-dependentes.

$\mathrm{O} \mathrm{pH}$, dentro do contexto farmacêutico, está diretamente relacionado com a solubilidade de substâncias, a estabilidade química e compatibilidade fisiológica com os tecidos orgânicos onde o fármaco irá agir, conforme TABELA 1, e a liberação controlada de fármacos (FERREIRA, 2002).

TABELA 1 - pH em algumas regiões e fluidos corporais.

\begin{tabular}{lclc}
\hline Região do corpo & $\mathbf{p H}$ & Fluidos ou secreções & $\mathbf{p H}$ \\
\hline Pele & $\sim 5,5$ & Líquido retal & $7,2-7,4$ \\
Rosto & $4,7-5,5$ & Lágrima & $\sim 7,4$ \\
Axilas & $6,1-6,8$ & Secreção nasal (adultos sadios) & $5,5-6,5$ \\
Cabelos & $\sim 5,0$ & Secreção nasal (crianças) & $5,0-6,7$ \\
Saco conjuntival & $7,3-8,0$ & Urina & 6,0 \\
Conduto auditivo & $6,0-7,8$ & Saliva & 6,9 \\
& & Sangue & 7,4 \\
Vaginal & $4,0-4,5$ & Perspiração (suor) & $\sim 5,0$ \\
& & Suco gástrico & $1,0-3,0$ \\
\hline
\end{tabular}

Dependendo do local de liberação do fármaco, o comportamento das micropartículas em diferentes pH's é um fator de extrema importância a ser investigado (THU, et al. 1996;GASEROD; SMIDSROD; SKJASK-BRAEK, 1998).

$\mathrm{O}$ azul de metileno tem peso molecular de $319,85 \mathrm{gmol}^{-1}$ e coeficiente de difusão livre em água a $2{ }^{\circ} \mathrm{C}$ de $8,3 \cdot 10^{-6} \mathrm{~cm}^{2} \mathrm{~s}^{-1}$ e $\lambda=655 \mathrm{~nm}$ e com baixo peso molecular permeia facilmente pelos poros de micropartículas de alginato sendo, portanto, um bom modelo para o estudo de liberação de substâncias com a mesma particularidade (KAMINSKI et al., 2009; DOHNAL; STEPANEK2010).

Este trabalho tem por objetivo avaliar o perfil de liberação do azul de metileno das micropartículas de alginato e reticuladas com quitosana, com ou sem a presença de sódio, em tampão pH 1,5, considerando o azul de metileno como modelo de princípio ativo de baixo peso molecular encapsulado.

\section{MATERIAL E MÉTODOS}

\subsection{MATERIAL}

Alginato de sódio (Protonal LF 20/40 FMC Biopolymer), Quitosana de alta densidade, viscosidade de 20 a 500 cps (Galena), Tween 80 (Henrifarma), Azul de 
metileno (Merck), Cloreto de cálcio (Merck), Reagentes Cloreto de sódio, Ácido clorídrico, Acido acético glacial e Cloreto de potássio (Synth) de grau analítico, Água deionizada(PERMUTION). Agitador de hélice IKA RW 20, Espectrofotômetro (Shimadzu UV-1800), Seringa (INJEX $5 \mathrm{ml}$ ), Agulha (BD 1,20 x $40 \mathrm{~mm}$ ) e Microscópio óptico (STUDAR).

\subsection{MÉTODOS}

As micropartículas de alginato-quitosana (MP1) foram preparadas a partir do gotejamento da fase 1 , composta por $0,5 \%$ de alginato de sódio e $2 \mathrm{~g}$ de solução aquosa de azul de metileno $1 \%$ sobre a fase 2 , composta por solução $1,0 \%$ de $\mathrm{CaCl}_{2}$, e $0,3 \%$ de quitosana previamente solubilizada em ácido acético $1 \%$. O gotejamento é feito em ângulo de $90^{\circ}$, a $6 \mathrm{~cm}$ de altura sob agitação à $200 \mathrm{rpm}$, utilizando uma seringa. A relação da fase 1 e fase 2 é de $1: 1(\mathrm{v} / \mathrm{v})$. O sistema permanece sob leve agitação de 70 rpm por 30 minutos. As micropartículas são recuperadas por tamização, eliminando-se todo excesso de água e pesadas para verificar o rendimento.

Adicionou-se aproximadamente $5 \mathrm{~g}$ de micropartículas em $500 \mathrm{~mL}$ de tampão ácido clorídrico de $\mathrm{pH}$ 1,5. A solução tampão foi doseada periodicamente por espectrofotometria no UV a $655 \mathrm{~nm}$ por $24 \mathrm{~h}$.

As micropartículas de alginato-quitosana-sódio (MP2) foram preparadas de forma idêntica às micropartículas (MP1) descritas anteriormente, com adição de cloreto de sódio à fase 2 na proporção de $1: 4(\mathrm{p} / \mathrm{p})$ de $\mathrm{CaCl}_{2}$ e $\mathrm{NaCl}$ respectivamente. $\mathrm{O}$ doseamento também foi realizado de forma idêntica as da (MP1).

Foi feita uma curva de calibração de azul de metileno de 7 pontos no espectrofotômetro e feita as medidas de absorbância das amostras em $\lambda=655 \mathrm{~nm}$.

\section{RESULTADOS E DISCUSSÃO}

Os métodos descritos neste trabalho produziram partículas entre 1 e $2 \mathrm{~mm}$, esféricas e com distribuição de tamanho homogênea. Dependendo da velocidade do agitador, posicionamento da seringa e altura da agulha sobre a fase 2, podem ocorrer deformações na morfologia das micropartículas, desde alongada até achatada. $\mathrm{O}$ tamanho das partículas depende principalmente da viscosidade do alginato e do calibre da agulha.

Diferentes métodos de formação de micropartículas resultam em variações na quantidade de fixação de quitosana, que influenciam nas propriedades físicas, permeabilidade e propriedades superficiais das mesmas. As micropartículas (MP1) de alginato de cálcio-quitosana foram feitas pelo processo de um estágio, no qual a solução de alginato de sódio é gotejada em solução de cálcio contendo quitosana. Este método apresenta maior capacidade de fixação da quitosana nas micropartículas, quando comparadas às de alginato-Ca mergulhadas, posteriormente, na solução de quitosana (GASEROD; SMIDSROD; SKJASK-BRAEK, 1998).

A presença de $\mathrm{Na}^{+}$na solução de $\mathrm{CaCl}_{2}$ no processo de obtenção das micropartículas (MP2), resultou num rendimento de produção cerca de $20 \%$ maior que o processo sem sódio (MP1). 
Segundo Gaserod, Smidsrod e Skjak-Braek (1998), a presença de íons $\mathrm{Na}^{+}$ na solução de $\mathrm{CaCl}_{2}$, favorece a formação de micropartículas mais homogêneas, que possuem maior capacidade de ligação com a quitosana durante a reticulação em 24 horas.

A quantidade de fixação de quitosana na reticulação tem se mostrado fortemente dependente do processo de formação das partículas. As micropartículas homogêneas exibem menor concentração de polímero na superfície, que as não-homogêneas, mostrando maior fixação de quitosana, em função do diâmetro maior dos poros obtidos na forma homogênea (GASEROD; SMIDSROD; SKJASK-BRAEK, 1998).

O tampão ácido clorídrico de $\mathrm{pH} 1,5$, simula o ambiente gástrico, permitindo analisar a liberação de princípios ativos, bem como o comportamento da micropartícula em si nesta condição. O perfil de liberação de azul de metileno é influenciado também com a presença do íon $\mathrm{Na}^{+}$na formação das micropartículas.

TU et al.(2005) realizaram o estudo das características de encapsulamento e liberação de micropartículas de alginato-Ca em meio ácido $\mathrm{pH} \mathrm{1,5,} \mathrm{simulando} \mathrm{fluido}$ gástrico e em água destilada, utilizando como modelo de substância hidrossolúvel, o azul de metileno, e a 4-fenilazoanilina como modelo de substância hidrofóbica.

$\mathrm{O}$ azul de metileno em fluido gástrico simulado $(0,1 \mathrm{~N} \mathrm{HCl})$ foi quase completa em 5 minutos, enquanto a liberação do 4-fenilazoanilina foi lenta em $0,1 \mathrm{~N} \mathrm{HCl}$.

Em água, a liberação do azul de metileno foi incompleta em água (65\%); observou-se a completa liberação na presença de $\mathrm{NaCl}$. A ordem de liberação do azul de metileno foi $\mathrm{HCl}>$ solução de $\mathrm{NaCl}>$ água. A liberação do azul de metileno é controlada por processo de difusão. A dissolução incompleta do azul de metileno das micropartículas de alginato em água pode ser em função da interação iônica com consequente manutenção da estrutura da micropartícula durante o tempo de estudo da liberação.

Dohnal e Stepanek (2010) avaliaram a liberação de azul de metileno e vitamina B12 de micropartículas de alginato-Ca em função da concentração de alginato. Os pesquisadores concluíram que o coeficiente de difusão do soluto depende da concentração do alginato no gel e das características deste alginato, como peso molecular e relação guluronato/manuronato na estrutura polimérica.

Concluiu que a capacidade de controlar tamanho e formato da microcápsula, combinado com o conhecimento da efetiva difusibilidade do soluto sobre uma concentração de alginato, pode ser usado para um design racional de microcápsulas, isto é, tomar decisões sobre formulação e parâmetros de processo necessários para adquirir um perfil de liberação requerido de uma determinada substância a ser encapsulada.

$\mathrm{O}$ azul de metileno foi escolhido para representar fármacos hidrofílicos de baixo peso molecular com $319.85 \mathrm{~g} / \mathrm{mol}$ (USP, 2009), por possuir metodologia de doseamento farmacopeica de aplicação prática e por facilitar a vizualização em bancada do seu encapsulamento.

O doseamento de azul de metileno na solução de reticulação (fase 2) apresentou valores de absorbância de 0,614 para MP1 e 0,627 para MP2, sugerindo um rendimento de encapsulamento semelhante, sendo o perfil de liberação mostrado no gráfico 1 que expressa as absorbâncias em função do tempo. 


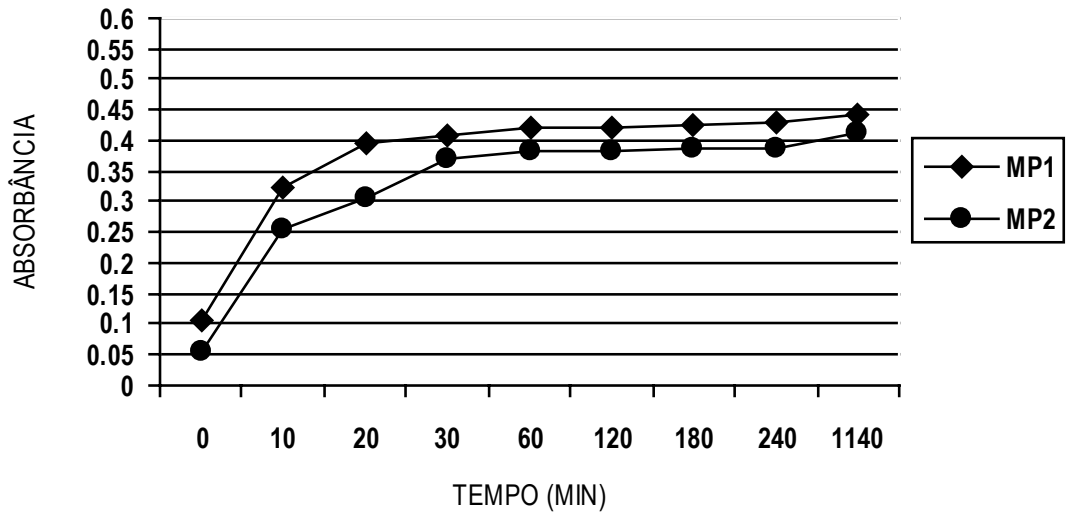

GRÁFICO 01 - Perfil de liberação de azul de metileno a partir de micropartículas de alginato-quitosana, com e sem adição de sódio.

A liberação do azul de metileno ocorreu de forma gradativa e sustentada, apresentando perfil muito semelhante entre os dois métodos. As micropartículas liberaram grande parte de seu conteúdo, em aproximadamente 30 minutos. Este tempo é superior ao obtido no trabalho de Tu et al. (2005), em que quase todo azul de metileno em meio ácido foi liberado em cerca de 5 minutos o que sugere que a reticulação com quitosana auxilia no processo de controle de liberação. Com adição de $\mathrm{NaCl}$ no meio ácido, perceberam aumento no tempo de liberação.

Os dados obtidos de literatura como o método de um estágio, a presença de sódio na solução reticulante, a porosidade da micropartícula, maior fixação de quitosana em presença de sódio, maior interação iônica, menor coeficiente de difusão, suportam o resultado obtido de MP2 e apresentado neste trabalho.

\section{CONCLUSÕES}

As microparticulas de alginato MP2, com adição de sódio e quitosana, mostraram-se promissoras para a liberação controlada de fármacos de baixo peso molecular em meio gástrico.

Para a otimização do processo de encapsulamento de fármacos de baixo peso molecular, deve-se buscar técnicas, concentrações e associações que prolonguem o tempo de contenção dos mesmos, melhorado de perfil de liberação em ambiente de $\mathrm{pH}$ ácido.

Os métodos utilizados neste trabalho mostraram ser uma alternativa para o desenvolvimento de formas farmacêuticas de liberação sustentada em pH extremo, como o encontrado no estômago, demonstrando que seu uso nessa faixa de $\mathrm{pH}$ pode ser proposto para o caso de fármacos de baixo peso molecular.

Sugere-se como próxima etapa de estudos a continuidade do aprimoramento de métodos de obtenção das micropartículas de alginato, utilizando a quitosana e $\mathrm{NaCl}$ para otimizar o perfil de liberação de substâncias de baixo peso molecular a serem encapsuladas. 


\section{REFERÊNCIAS}

ALLEN JR, L. V.; POPOVICH, N. G.; ANSEL, H. C. Formas farmacêuticas e sistemas de liberação de fármacos. 8. ed. Porto Alegre: Artmed, 2007.

CORRÊA, R. M. Produção de micropartículas por gelificação iônica para alimentação de larvas de peixe: estudos de sistema-modelo com inclusão de micropartículas lipídicas ou emulsão lipídica e testes in vivo. 151f. Tese (Doutorado em Alimentação e Nutrição) - Faculdade de Engenharia de Alimentos, Universidade Estadual de Campinas, Campinas, 2008.

DARRABIE, M. D.; KENDALL, W. F.; OPARA, E. C. Effect of alginate composition and gelling cation om microbead swelling. Journal of Microencapsulation, v. 23, n. 06, p. 613-621, 2006.

DOHNAL, J.; STEPANEK, F. Inkjet fabrication and characterization of calcium alginate microcapsules. Powder Technology, v. 200, p. 254-259, 2010.

FERREIRA, A. O. Guia prático de farmácia magistral. São Paulo: Pharmabooks, 2002.

FUNDUEANU, G.; ESPOSITO, E.; MIHAI, D.; CARPOV, A.; DESBRIERES, J.; RINAUDO, M.; NATRUZZI, C. Preparation and characterization of Ca-alginate microspheres by a new emulsification method. International Journal of Pharmaceutics, v. 170, p. 11-21, 1998.

GASSEROD, O.; SMIDSROD, O.; SKJASK-BRÑK, G. Microcapsules of alginate-chitosan - I A quantitative study of the interaction between alginate and chitosan. Biomaterials, v.19. p.1815-1825. 1998.

HAN, M. R.; KWON, M. C.; LEE, H. Y.; KIM, J. C.; KIM, J. D.; YOO, S. K.; SIN, I. S.; $\mathrm{KIM}$, S. M. pH-dependent release property of alginate beads containing calcium carbonate particles. Journal of Microencapsulation, v. 24, n. 8, p. 787-796, 2007.

KAMINSKI, G. A. T.; SILVÉRIO, L. C.; PASQUALIM, P.; COSTA, C. K.; ZANIN, S. M. W. Avaliação da influência da variabilidade de óleos no microencapsulamento de fármacos hidrofílicos de baixo peso molecular por emulsificação a/o. Visão Acadêmica, vol. 11. 2009.

LIRA, A. A. M.; ROSSETTI, F. C.; NANCLARES, D. M. A.; FEDERMAN NETO, A.; BENTLEY, M. V. L. B.; MARCHETTI J. M. Preparation and characterization of chitosan-treated alginate microparticles incorporating all-trans retinoic acid. Journal of Microencapsulation, v. 26, n. 3, p. 243-250, 2009.

LÓPEZ, C. R.; ALONSO, M. J. Microencapsulacion. In: VILA JATO, J. L. Tecnología farmacéutica I: aspectos fundamentales de los sistemas farmacéuticos y operacio- 
nes básicas. Madrid: Sintesis, 2001. p. 577-608.

LOURENÇO, V. A. Desenvolvimento e avaliação de micropartículas de quitosana para veiculação de dimetiletanol (DMAE) na pele. 117p. Dissertação (Mestrado em Ciências Farmacêuticas) - Faculdade de Ciências Farmacêuticas, Universidade de São Paulo, Riberão Preto, 2006.

SANTOS, A. B.; FERREIRA, V. P.; GROSSO, C. R. F. Microcápsulas: uma alternativa viável. Biotecnologia, Ciência e Desenvolvimento, Brasília, ano 3, n. 16, set./out. 2000

SCHOUBBEN, A.; BLASI, P.; GIOVAGNOLI, S.; ROSSI, C.; RICCI, M. Development of a scalable procedure for fine calcium alginate particle preparation. Chemical Engineering Journal, 2010, doi:10.1016/j.cej.2010.02.062.

STORPIRTIS, S.; GONÇALVES, J. E.; CHIANN, C.; GAI, M. N. Biofarmacotécnica. Rio de Janeiro: Guanabara-Koogan, 2009.

SUAVE, J.; DALL'AGNOL, E. C.; PEZZIN, A. P. T.; SILVA, D. A. K.; MEIER, M. M.; TAN, L. H.; CHAN, L. W.; HENG, P. W. S. Alginato/starch composites as wall material to achieve microencapsulation with high oil loading. Journal of Microencapsulation, v. 26, n. 03, 263-271, 2009.

THU, B.; BRUHEIM, P.;ESPEVIK, T.; SMIDSROD, P.; SOON-SHIONG, G.; SKJAKBRAEK, G. Alginate polycation microcapsules. II. Some functional properties. Biomaterials, n.17. p.1069-1079, 1996.

TU, J. BOLLA, S.; BARR, J.; MIEDEMA, J.; LI, X.; JASTI, B. Algiante microparticles prepared by spray-coagulation method: preparation, drug loading and release characterization. International Journal of Pharmaceutics, v. 303, p. 171-181, 2005.

UNITED STATES PHARMACOPOEIA, 32ª edição, 2009. 